\title{
Patterns of Drug Utilization in a Neonatal Intensive Care Unit
}

\author{
Indulekha Warrier, $M D$, Wei Du, PhD, Girija Natarajan, $M D$, \\ Vali Salari, PhD, and Jacob Aranda, MD, PhD
}

The objective of this study was to determine drug use in newborns at an inborn tertiary care neonatal intensive care unit, serving a predominantly African American population, to identify educational/research priorities in neonatal drug therapy. Data on demographics and exposure rates to all drugs from 6839 neonates born between January 1997 and June 2004 were analyzed. Number of drugs used was correlated with race, gender, gestational age, birthweight, and survival status. The contribution of these factors to mean drug use was predicted by multivariate regression analysis. In this population of $80 \%$ African Americans, mean drug use was 3.6/infant, with the highest use in the 24- to 27-week gesta- tional age group (11.7/infant). Ampicillin and cefotaxime had the highest exposure rates. Premature infants had high use of surfactant, pressor agents, and diuretics. Caucasians, males, gestational age $<28$ weeks, and birthweight $<1000 \mathrm{~g}$ were the risk factors for higher drug exposure. Future research/ education must emphasize these therapeutic areas with priority assigned to low-birthweight infants.

Keywords: Drug utilization; neonate; neonatal intensive care unit

Journal of Clinical Pharmacology, 2006;46:449-455 C2006 the American College of Clinical Pharmacology
$\mathbf{M}$ any advances have been made in the area of pediatric clinical pharmacology, ${ }^{1-3}$ yet a paucity of information to guide rational prescribing in children and newborns remains. This is a problem faced by practitioners in the United States as well as around the world, leading to reliance on suboptimal prescribing data placing newborns and children at risk of high rates of adverse events. ${ }^{4-7}$ A 1994 study found that there was a substantial lack of epidemiological studies related to drug utilization in children, relegating them to the status of "methodologic orphans." "A current search of literature in the area of pediatric pharmacoepidemiology indicates that the problem continues to persist, with very few studies being published in this field.

This issue is all the more important in the neonatal population. Newborns who are products of high-risk pregnancies, premature infants, or those with both

From the Division of Pediatric Clinical Pharmacology (Dr Warrier, Dr Du, Dr Aranda) and Division of Neonatology (Dr Natarajan, Dr Salari, Dr Aranda), Children's Hospital of Michigan, Wayne State University, Detroit, Michigan. Submitted for publication September 1, 2005; revised version accepted December 3, 2005. Address for reprints: Indulekha Warrier, MD, Fellow, Division of Clinical Pharmacology, 3N47, Children's Hospital of Michigan, 3901 Beaubien, Detroit, MI 48201; e-mail: induw@ med.wayne.edu.

DOI: $10.1177 / 0091270005285456$ risk factors are at greater risk for medication exposure. Published data, albeit scarce, show that the number of drugs administered varies inversely with the gestational age and/or birthweight of the infant. ${ }^{8-12}$ Fetal maturity also plays a role in determining the pharmacokinetics of drugs. This is due to developmental factors affecting (1) drug absorption (in the case of orally administered drugs); (2) drug distribution such as body compartment sizes/total body water content, protein binding, hemodynamic factors, and so on; and (3) drug metabolism/clearance (either renal or hepatic) due to ontogenetic differences in blood flow through the metabolizing organs as well as drug-metabolizing enzyme activity. ${ }^{2,3,13}$ Thus, newborn infants are more prone to drug adverse events when compared to adults. ${ }^{14}$ Exposure to multiple drugs is also known to be a factor leading to increased incidence of adverse events in neonates. ${ }^{15,16}$

Published data show that the average number of drugs administered per infant in the neonatal intensive care unit (NICU) had progressively increased over the years. A Canadian study done in the 1970s showed an increase in mean drug per neonate from 3.4 to 6.2 over a 3-year time period. ${ }^{17}$ The mean number of drugs used in newborns in the NICU in an Australian study published in 1989 was $8.6 \pm 0.9 .^{18}$ The median number of 
drugs used per infant in a US population published during this same time period was $8 /$ infant. ${ }^{10}$ In most of these studies, antibiotics were the one category of drugs that had maximum utilization rates. There are no data available on the prevalence of antifungal use in this population, despite the recognition of the increasing incidence of fungal infections and subsequent antifungal therapy in newborns. ${ }^{19-23}$

Changes in neonatal drug therapy, including the introduction of many new drugs, have occurred over the past decade. There have been few studies documenting these changes, and an ongoing review of drug utilization patterns in sick newborns was warranted to assign research and educational priorities in neonatal pharmacology. Our study objectives were to (1) determine the actual drug utilization patterns in neonates, (2) identify the most commonly used drugs in neonates and the actual frequency of their use, and (3) identify the areas in neonatal pharmacology that are in need of further research, as reflected by our experience at a single, tertiary care neonatal intensive care unit.

\section{MATERIALS AND METHODS}

\section{Study Design and Population}

This study was approved by the Wayne State University Human Investigations Committee and was a retrospective data analysis of the drug utilization patterns of 6839 neonates treated at Hutzel Women's Hospital (HWH) in Detroit between January 1997 and June 2004. The neonates included in this study had to be born between January 1997 and June 2004, with postnatal age $\leq 28$ days at admission, and required the administration of at least 1 drug. Both male and female infants of any race admitted to the Hutzel NICU or Progressive Care Nursery (PCN-a step-down unit of NICU) were included. HWH is a large teaching hospital that serves as the obstetrical site for a predominantly African American urban population, and the NICU is a level III unit that provides care for an almost exclusively inborn population.

\section{Data Sources}

Study data were obtained from a review of the neonatal database (NEOSYSTEM), a network-based computer system designed and in use at $\mathrm{HWH}$ since January 1997. The purpose of this system is to generate admission notes, daily progress notes, discharge summaries, and monthly statistics for neonates admitted to the NICU, PCN, and normal newborn units. PC (personal computer) clients connected to the main server are lo- cated at these 3 units. Residents, staff physicians, and nursing staff who provide care for the infants are responsible for data entry. FoxPro software was used to retrieve data.

\section{Study Endpoints and Analysis}

Data on gender, race, gestational age, birthweight, length of hospital stay (number of days from date of birth until date of discharge), survival status at discharge, and all pharmacological agents used were collected and analyzed. Data regarding the following were not collected: blood and blood products (except fresh frozen plasma), total parenteral nutrition, oxygen administration, vitamin $\mathrm{K}$ prophylaxis, erythromycin ophthalmic prophylaxis, routine cord care, and any vaccinations received. We also did not assess the utilization rates of routine parenteral fluids. Normal saline was included in our analysis only when it was given for a specific indication of hypotension.

Drugs used were classified into 10 categories: antibiotics, diuretics, vitamins/minerals/nutritionals, biologicals, cardiovascular agents, respiratory agents, ophthalmic agents, neurological agents, hormones, and miscellaneous agents. The total number of drugs administered to each neonate was calculated and tabulated, and exposure rates to all drugs used were examined.

Race, gender, gestational age, birthweight, and discharge status were then correlated with the number of drugs used with $t$ tests, Pearson correlation analysis, or analysis of variance. Multiple regression analysis was also performed on the number of drugs used with race, gender, discharge status, birthweight, and gestational age as predictors.

\section{RESULTS}

As shown in Table I, ethnicity was predominantly African American, with Caucasians, Hispanics, and other minorities represented in smaller numbers. Of these, $46 \%$ were females and $54 \%$ were males. Mean length of stay was $15 \pm 24$ (SD) days, mean gestational age was $35 \pm 5$ (SD) weeks, and mean birth weight was $2498 \pm$ 1000 (SD) grams. Approximately 97\% of babies were alive at the time of discharge.

Mean drug use was 3.6 \pm 3.9 (SD) per infant when all infants were included, although the average number of drugs administered varied depending on gestational age and birthweight. The highest drug use was in the 24- to 27-week gestational age infants at 11.8/infant (Fig. 1 ), followed by the <23-week category at 9.9/infant. When based on birthweight, the highest mean drug use 
Table I Population Characteristics

\begin{tabular}{lc}
\hline \hline Ethnicity, \% & \\
African American & 79.95 \\
Caucasian & 13.85 \\
Hispanic & 3.22 \\
Other & 2.96 \\
Gender, $\%$ & 53.78 \\
$\quad$ Male & 46.22 \\
Female & 2498 (SD $=1000$ g) \\
Mean birthweight & 35 (SD $=5$ weeks) \\
Mean gestational age & \\
Percentage of infants in each & 0.5 \\
gestational age category & 11.7 \\
$\quad \leq 23$ weeks & 31.8 \\
24 to $\leq 27$ weeks & 48 \\
28 to $\leq 31$ weeks & 15 (SD = 24 days) \\
32 to $\leq 37$ weeks & 97 \\
$\quad \geq 38$ weeks & \\
Mean length of stay & \\
Survival rate, $\%$ & \\
\hline & a. Mean length of stay: number of days from date of birth until date of \\
discharge. & \\
b. Survival rate: whether discharged alive or not. If the patient expired, \\
then date of death was the same as date of discharge.
\end{tabular}

was in the 501- to 750-g birthweight category at 12.3/ infant (Fig. 2), followed by the 751- to 1000-g infant at $11.1 /$ infant. Infants with birthweight $<500 \mathrm{~g}$ had mean drug use of 8.8/infant.

The top 15 drugs used in all infants are listed in Table II. The antibiotics ampicillin and cefotaxime were the 2 most widely used drugs, with rates of exposure far outstripping exposure to any other drug. The third most widely used drug was surfactant (Survanta), which was probably a reflection of the high percentage of premature infants (52\%, Table I). Exposure to the next 13 drugs on the list was less than a quarter of that of the top 2 drugs.

A subgroup analysis by gestational age revealed an interesting but not unexpected progression in the use of certain drugs (Table III). All infants of gestational age $\leq 23$ weeks were exposed to ampicillin, cefotaxime, and surfactant. Exposure to these drugs decreased in the 24to 27-week infants and continued to drop as the gestational age increased. But in the case of vancomycin, iron, diuretics, dexamethasone, indomethacin, and respiratory agents other than surfactant, exposure rates peaked in the 24- to 27-week infants and subsequently decreased. In fact, other than the antibiotics ampicillin and cefotaxime, which did not have a significant decrease in exposure across gestational age groups, all other drugs had a marked decrease in exposures beyond the 27-week age group.

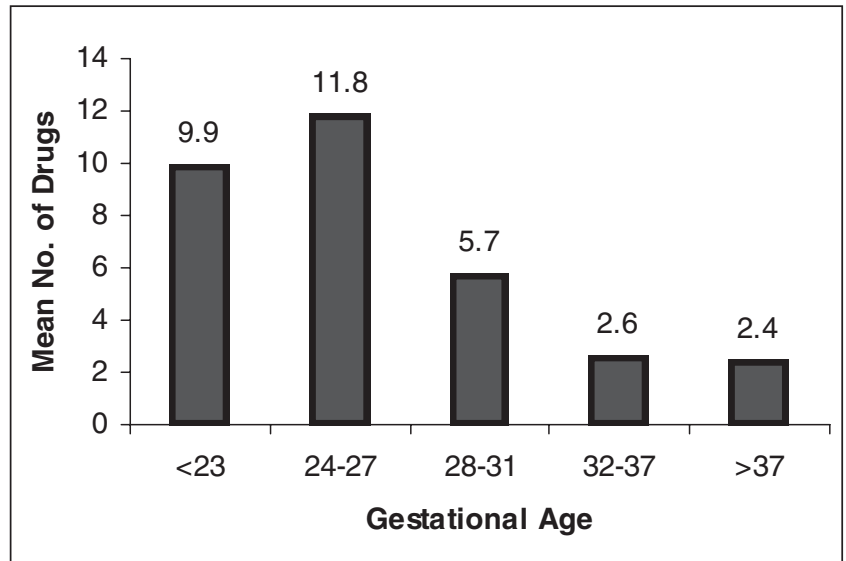

Figure 1. Mean drug use by gestational age.

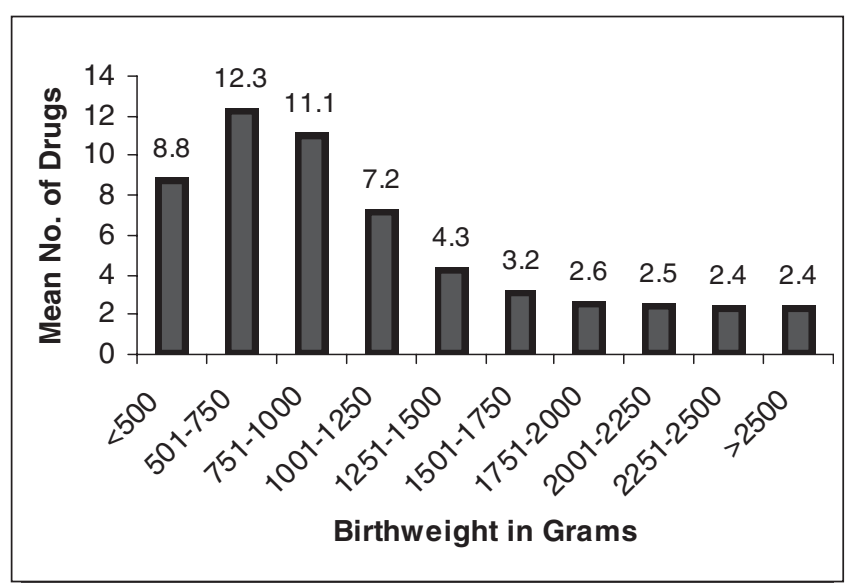

Figure 2. Mean drug use by birthweight.

Antifungal exposure (including amphotericin deoxycholate, liposomal amphotericin, fluconazole, flucytosine, and nystatin) was $2.3 \%$ in all infants and was highest in the $<23$-week infants at $20.6 \%$, followed by 24 - to 27 -week infants at $19 \%$. In the 28 - to 31 -week infants, it decreased drastically to $5.6 \%$ and was $<1 \%$ for infants $>32$ weeks. Most of this was contributed to by amphotericin (both liposomal and deoxycholate), with exposure rates being $17.7 \%, 18.3 \%, 5 \%$, and $<1 \%$ in infants $<23$ weeks, 24 to 27 weeks, 28 to 31 weeks, and $>32$ weeks, respectively.

A multivariate analysis of the data was performed to identify factors associated with higher number of drug use (Table III). Caucasian ethnicity, male gender, a gestational weight of $<28$ weeks, and weight $<1000$ g were associated with higher drug use, whereas early death 
Table II Exposure Rates for the Top 15 Drugs: All Infants

\begin{tabular}{lc}
\hline \hline Name of Drug & \% of Infants Exposed \\
\hline Ampicillin & 94.22 \\
Cefotaxime & 92.24 \\
Survanta & 18.18 \\
Plasmanate & 11.73 \\
Dopamine & 10.05 \\
Iron & 9.50 \\
Vancomycin & 8.79 \\
Theophylline & 6.61 \\
Caffeine & 5.80 \\
Aldactone & 5.51 \\
Dobutamine & 5.32 \\
Diuril & 5.31 \\
Furosemide & 5.28 \\
Calcium supplement & 5.26 \\
Penicillin & 5.06
\end{tabular}

( $<7$ days) was associated with lower drug use. Although gestational age and birthweight are known to be correlated, a regression model including both factors yielded a higher $R^{2}(0.502)$ than models including only 1 of the 2 factors ( 0.445 and 0.454 ). Thus, we chose to include both factors in our final model to maximize the predictive power.

\section{DISCUSSION}

Recent advances in prenatal care have resulted in an increase in drug exposure rates in pregnant mothers, causing infants to be exposed to a multitude of different drugs, even when in utero. ${ }^{24,25}$ Advances in medical technology have also resulted in improved survival rates in both term and preterm infants, including highrisk and low-birthweight infants. ${ }^{26}$ As a result of these changes in the medical management of the newborn, drug exposure patterns in infants have also changed. Although there is a general appreciation that neonates, especially preterm neonates, have high drug utilization rates, a systematic evaluation is needed to prioritize areas in need of further pharmacotherapeutic research. This will also help document changes in the trend of medication use, define the groups of infants that are at higher risk for adverse events, and provide necessary information to estimate the costs and benefits of current NICU care.

In this study, we examined an urban, medically underserved population with a high incidence of highrisk pregnancies. The NICU/PCN studied is a desig- nated level III center and primarily admits infants delivered by the HWH obstetrical service. We excluded data on the use of vitamin K prophylaxis, erythromycin ophthalmic prophylaxis, and routine cord care as these are administered to all infants born at $\mathrm{HWH}$, and data on these would not have contributed any significant information. Data regarding parenteral solutions other than normal saline given for an indication of hypotension, blood and blood products except fresh frozen plasma, total parenteral nutrition, oxygen administration, and any vaccinations received were also not collected because of their extensive use.

The population characteristics of our patients differed from those of previous studies in that it consisted of a majority of African Americans, a historically underserved community. The mean gestational age and birthweight were also higher than those of previous studies. Mean drug use in our study was lower than we expected, and when compared to previous studies, there was no increase in mean drug use. ${ }^{11,18}$ This could be explained by the fact that (1) the patients in our study had greater mean gestational age and birthweight, (2) our NICU cares for patients with lesser disease acuity, and (3) previous studies included information regarding some of the drugs that we excluded due to the ubiquity of their use. Comparison with previous studies was thus difficult due to these reasons as well as the temporal distance involved.

Very premature infants are known to have high rates of medical complications necessitating prolonged NICU stay periods, which ultimately result in higher mean drug use and exposure rates. Our results were consistent with this, with mean drug use being maximal in the 24- to 27-week infants, with the $\leq 23$-week infants a close second. It is also interesting to note that similar to previous studies, in our study also, ethnicity and gender played a role in determining survival outcomes in neonates, with African Americans and females having better survival outcomes (data not shown). ${ }^{26}$ It is known that Caucasian neonates and male neonates have higher mortality rates. ${ }^{26,27} \mathrm{Al}-$ though it is not possible to derive any firm conclusions, our observed increase in drug exposure in these groups could be a reflection of an increased morbidity in these groups.

The high rate of antibiotic exposure in our study is similar to studies published in the past ${ }^{9}$ and is probably due to the standard practice of administering antibiotics pending bacterial culture results in sick neonates and is not a true reflection of the incidence of bacterial infection. ${ }^{27}$ Vancomycin exposure was also surprisingly high in our study when compared to the actual incidence of methicillin-resistant organisms in the NICU 


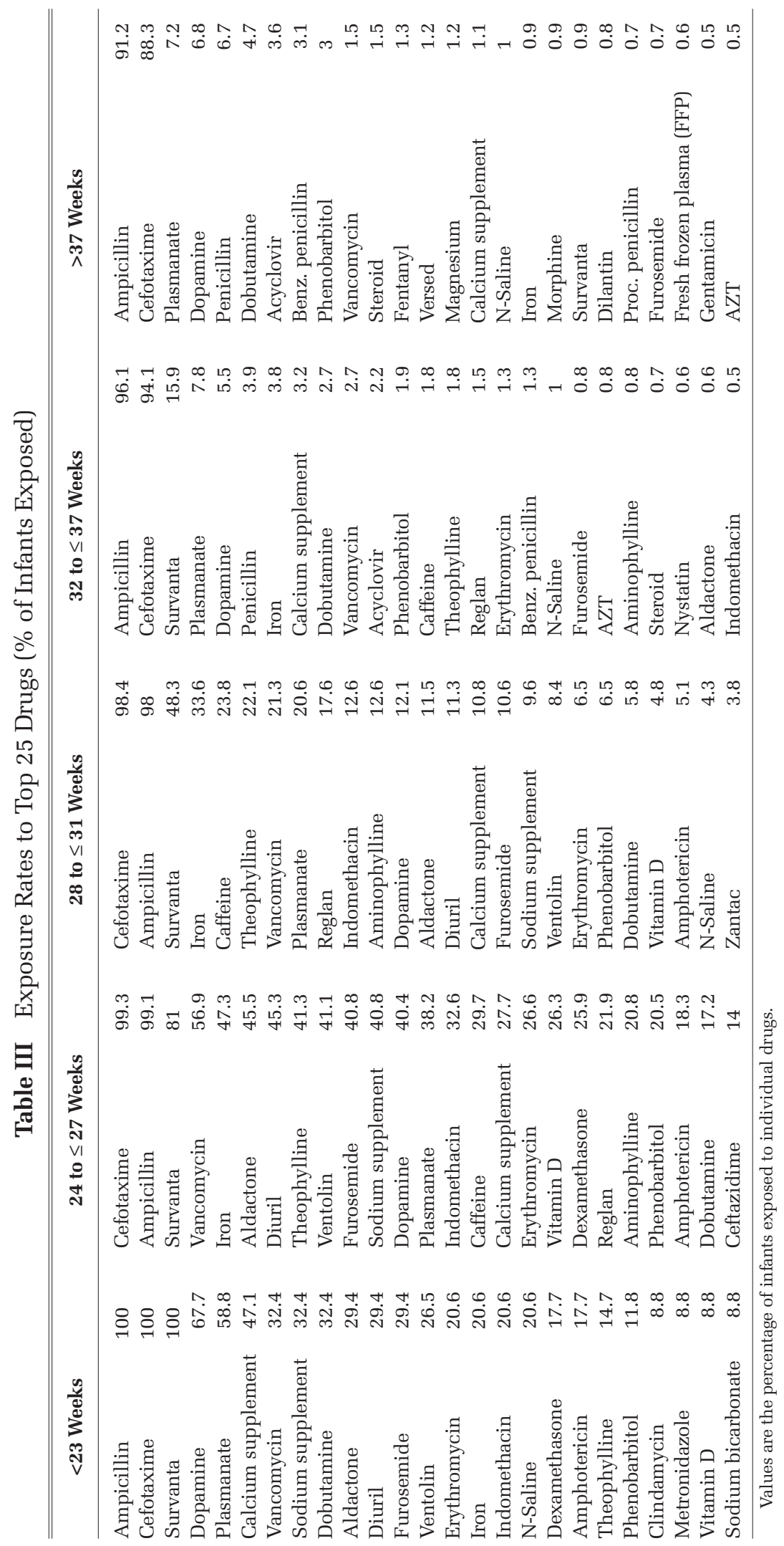


Table IV Mean Drug Use per Infant

\begin{tabular}{lccc}
\hline \hline Drug Classes & Mean & Range & $\begin{array}{c}\text { Standard } \\
\text { Deviation }\end{array}$ \\
\hline Total drug & 3.64 & $1-36$ & 3.89 \\
Antibiotics & 2.21 & $0-11$ & 0.93 \\
$\quad$ Antibacterials & 2.14 & $0-9$ & 0.78 \\
$\quad$ Antifungals & 0.04 & $0-4$ & 0.24 \\
$\quad$ Antivirals & 0.04 & $0-2$ & 0.19 \\
Respiratory & 0.44 & $0-9$ & 1.07 \\
Supportive & 0.27 & $0-9$ & 0.78 \\
Chorionic villus & & & \\
$\quad$ sampling (CVS) & 0.21 & $0-5$ & 0.59 \\
Diuretics & 0.16 & $0-4$ & 0.64 \\
Miscellaneous & 0.15 & $0-3$ & 0.4 \\
Central nervous system & 0.09 & $0-6$ & 0.38 \\
Gastrointestinal & 0.06 & $0-3$ & 0.29 \\
Biologicals & 0.02 & $0-2$ & 0.15 \\
Hormone & 0.02 & $0-2$ & 0.16 \\
Ophthalmic & 0.01 & $0-1$ & 0.07 \\
\hline \multicolumn{2}{l}{ Drug classification is based on the following source: Young TE, Mangani B. } \\
$\quad$ Neofax Manual. 17th ed. Battle Creek, Mich: Acorn Publishing; 2004.
\end{tabular}

Table V Factors Affecting the Risk for Drug Exposure

\begin{tabular}{lcr}
\hline \hline Variable & $\boldsymbol{\beta}$ & $\boldsymbol{P}$ Value \\
\hline Caucasian ethnicity & 0.41 & $<.0001$ \\
Male gender & 0.25 & .0003 \\
Gestational age $>28$ weeks & -5.7 & $<.0001$ \\
Birthweight $>1000$ g & -5.21 & $<.0001$ \\
Early death (length of stay $<7$ days) & -5.17 & $<.0001$ \\
\hline
\end{tabular}

$\beta=$ regression coefficient. The negative regression coefficients show that mean drug exposure had an inverse relationship with indicated parameters.

as reported in literature. ${ }^{28-30}$ Exposure to amphotericin paralleled the incidence of neonatal Candida infection reported in literature. ${ }^{21-23}$ It was not surprising that surfactant and other respiratory agents, vasopressors, and diuretics had high exposure rates, especially in the very premature infant, although the high exposure to iron supplements, sodium supplements, metoclopramide, and indomethacin was unexpected. Pressors had high exposure rates in the very premature infant, although these drugs have not been well studied in this population. ${ }^{31}$ Diuretic use is also high in the premature infant, although long-term outcomes of diuretic therapy are still unknown. ${ }^{32}$ Individual agents used have also changed, with drugs such as kanamycin, tolazoline, having disappeared from the formulary and newer drugs such as surfactant, ceftazidime, cefepime, making their presence felt.

There have been very few studies documenting the observed changes and comparisons across gestational age groups, making this study pertinent. Such documentation is important, as neonates are more prone to adverse events/potential adverse events. ${ }^{33}$ This may be due to polypharmacy, which is known to increase the risk of adverse events; ${ }^{34,35}$ lack of proper drug evaluation trials; and, in the case of premature/lowbirthweight infants, immaturity of drug-metabolizing pathways. It also helps define the extent of expenditure due to pharmacotherapy that is driven by the different gestational ages.

A strength of our study is that it is the largest of its kind ( $\mathrm{n}=6839$ ), thus increasing the power and validity of the conclusions drawn. A limitation was that quality of data collection was dependent on the personnel entering the data. Because data were entered by different individuals (residents, fellows, nursing staff), there may have been some data entry deficiencies, leading to uneven reporting of drug use. This may have resulted in a systematic underestimation of drugs administered. Data regarding analgesic use may have been underrepresented because they are given on an asneeded basis and may not always be entered into the system. In summary, it is clear that the trend toward polypharmacy continues in neonates in the NICU, and drug exposure continues to be dictated by gestational age and/or birthweight. There still remain many areas in neonatal pharmacology that are in need of further studies, especially with regard to the use of antibiotics, pressor agents, and diuretics in the premature infant.

This study was supported by the Sarnaik Pediatric Research for Residents and Fellows Endowment, and the Children's Research Center of Michigan.

\section{REFERENCES}

1. Roberts R, Rodriguez W, Murphy D, Crescenzi T. Pediatric drug labeling: improving the safety and efficacy of pediatric therapies. JAMA. 2003;290:905-911.

2. Hines RN, McCarver DG. The ontogeny of human drug-metabolizing enzymes: phase I oxidative enzymes. J Pharmacol Exp Ther. 2002;300:355-360.

3. McCarver DG, Hines RN. The ontogeny of human drug-metabolizing enzymes: phase II conjugation enzymes and regulatory mechanisms. J Pharmacol Exp Ther. 2002;300:361-366.

4. Ten Eick AP, Reed MD. Clinical pharmacology research in the pediatric patient: the challenge continues. Prog Pediatr Cardiol. 2000; 12:29-35.

5. Choonara I. Unlicensed and off-label drug use in children: implications for safety. Expert Opin Drug Saf. 2004;3:81-83. 
6. Assael BM. Therapeutic orphans: European perspective. Pediatrics. 1999;104(Pt 2):591-592.

7. Survey of the current state of pediatric drug use in Japan (1994-6). Drug Therapy Committee of the Japan Pediatric Society. Pediatr Int. 2000;42:109-113.

8. Bonati M. Epidemiologic evaluation of drug use in children. JClin Pharmacol. 1994;34:300-305.

9. Gortner L. Drug utilisation in preterm and term neonates. Pharmacoeconomics. 1993;4:437-445.

10. Lesko SM, Epstein MF, Mitchell AA. Recent patterns of drug use in newborn intensive care. J Pediatr. 1990;116:985-990.

11. Aranda JV, Cohen S, Neims AH. Drug utilization in a newborn intensive care unit. J Pediatr. 1976;89:315-3l7.

12. Early neonatal drug utilization in preterm newborns in neonatal intensive care units. Italian Collaborative Group on Preterm Delivery. Dev Pharmacol Ther. 1988;11:1-7.

13. Blumer JL, Reed MD. Principles of neonatal pharmacology. In: Yaffe SJ, Aranda JV, eds. Neonatal and Pediatric Pharmacology. 3rd ed. Baltimore: Lippincott, Williams \& Wilkins; 2005:146-158.

14. van den Anker JN. Managing drugs safely. Semin Fetal Neonatal Med. 2005;10:73-81.

15. Knight M. Adverse drug reactions in neonates. J Clin Pharmacol. 1994;34:128-135.

16. Aranda JV. Factors associated with adverse drug reactions in the newborn. Pediatr Pharmacol 1983;3:245-249.

17. Aranda JV, Clarkson S, Collinge JM. Changing pattern of drug utilization in a neonatal intensive care unit. Am J Perinatol. 1983;1:2830 .

18. Daniell AJ, Darlow BA. Audit of drug usage in a regional neonatal intensive care unit. Aust Paediatr J. 1989;25:207-210.

19. Kaufman D, Fairchild KD. Clinical microbiology of bacterial and fungal sepsis in very-low-birth-weight infants. Clin Microbiol Rev. 2004;17:638-680.

20. Roilides E, Farmaki E, Evdoridou J, et al. Neonatal candidiasis: analysis of epidemiology, drug susceptibility, and molecular typing of causative isolates. Eur JClin Microbiol Infect Dis. 2004;23:745-750.

21. Kossoff EH, Buescher ES, Karlowicz MG. Candidemia in a neonatal intensive care unit: trends during fifteen years and clinical features of 111 cases. Pediatr Infect Dis J. 1998;17:504-508.

22. Stratov I, Gottlieb T, Bradbury R, O'Kane GM. Candidaemia in an Australian teaching hospital: relationship to central line and TPN use. J Infect. 1998;36:203-207.
23. Johnsson $H$, Ewald U. The rate of candidaemia in preterm infants born at a gestational age of 23-28 weeks is inversely correlated to gestational age. Acta Paediatr. 2004;93:954-958.

24. Glover DD, Amonkar M, Rybeck BF, Tracy TS. Prescription, overthe-counter, and herbal medicine use in a rural, obstetric population. Am J Obstet Gynecol. 2003;188:1039-1045.

25. Henry A, Crowther C. Patterns of medication use during and prior to pregnancy: the MAP study. Aust N Z J Obstet Gynaecol. 2000; 40:165-172.

26. Alexander GR, Kogan M, Bader D, Carlo W, Allen M, Mor J. US birth weight/gestational age-specific neonatal mortality: 1995-1997 rates for whites, Hispanics, and blacks. Pediatrics. 2003;111:e61-6.

27. Stevenson DK, Verter J, Fanaroff AA, et al. Sex differences in outcomes of very low birthweight infants: the newborn male disadvantage. Arch Dis Child Fetal Neonatal Ed. 2000;83:F182-F185.

28. Spitzer AR, Kirkby S, Kornhauser M. Practice variation in suspected neonatal sepsis: a costly problem in neonatal intensive care. $J$ Perinatol. 2005;25:265-269.

29. Toledano H, Schlesinger Y, Raveh D, et al. Prospective surveillance of vancomycin-resistant enterococci in a neonatal intensive care unit. Eur J Clin Microbiol Infect Dis. 2000;19:282-287.

30. Karlowicz MG, Buescher ES, Surka AE. Fulminant late-onset sepsis in a neonatal intensive care unit, 1988-1997, and the impact of avoiding empiric vancomycin therapy. Pediatrics. 2000;106:13871390.

31. Dasgupta SJ, Gill AB. Hypotension in the very low birthweight infant: the old, the new, and the uncertain. Arch Dis Child Fetal Neonatal Ed. 2003;88:F450-F454.

32. Brion LP, Primhak RA, Ambrosio-Perez I. Diuretics acting on the distal renal tubule for preterm infants with (or developing) chronic lung disease. Cochrane Database Syst Rev. 2002;1:CD001817.

33. Kaushal R, Bates DW, Landrigan C, et al. Medication errors and adverse drug events in pediatric inpatients. JAMA 2001;285:21142120.

34. Gonzalez-Martin G, Caroca CM, Paris E. Adverse drug reactions (ADRs) in hospitalized pediatric patients: a prospective study. Int $J$ Clin Pharmacol Ther. 1998;36:530-533.

35. Martinez-Mir I, Garcia-Lopez M, Palop V, Ferrer JM, Rubio E, Morales Olivas FJ. A prospective study of adverse drug reactions in hospitalized children. Br J Clin Pharmacol. 1999;47:681-688. 